\title{
Metabolic Analysis of the Asparagine and Glutamine Dynamics in an Industrial CHO Fed-Batch Process
}

\author{
Brian Kirsch ${ }^{1}$, Sandra Bennun ${ }^{2}$, Adam Mendez ${ }^{1}$, Amy Johnson ${ }^{2}$, Hongxia Wang $^{2}$, Haibo \\ $\mathrm{Qiu}^{2}$, Ning $\mathrm{Li}^{2}$, Shawn Lawrence ${ }^{2}$, Hanne $\mathrm{Bak}^{2}$, and Michael Betenbaugh ${ }^{1}$ \\ ${ }^{1}$ Johns Hopkins University \\ ${ }^{2}$ Regeneron Pharmaceuticals Inc
}

January 25, 2021

\begin{abstract}
Chinese Hamster Ovary $(\mathrm{CHO})$ cell lines are grown in cultures with varying asparagine and glutamine concentrations, but further study is needed to characterize the interplay between these amino acids. By following 13C-glucose, 13C-glutamine, and 13C-asparagine tracers using metabolic flux analysis (MFA), CHO cell metabolism was characterized in an industrially relevant fed-batch process under glutamine supplemented and low glutamine conditions during early and late exponential growth. For both conditions MFA revealed glucose as the primary carbon source to the tricarboxylic acid (TCA) cycle followed by glutamine and asparagine as secondary sources. Early exponential phase CHO cells prefer glutamine over asparagine to support the TCA cycle under the glutamine supplemented condition, while asparagine was critical for TCA activity for the low glutamine condition. Overall TCA fluxes were similar for both conditions due to the trade-offs associated with reliance on glutamine and/or asparagine. However, glutamine supplementation increased fluxes to alanine, lactate and enrichment of glutathione, N-Acetyl-Glucosamine (NAG) and pyrimidine-containing-molecules. The late exponential phase exhibited reduced central carbon metabolism dominated by glucose, while lactate reincorporation and aspartate uptake were preferred over glutamine and asparagine. These $13 \mathrm{C}$ studies demonstrate that metabolic flux is process time dependent and can be modulated by varying feed composition.
\end{abstract}

\section{Hosted file}

Dynamics of Asparagine and Glutamine - Kirsch - Main Body - Jan2021.pdf available at https://authorea.com/users/391643/articles/505690-metabolic-analysis-of-the-asparagineand-glutamine-dynamics-in-an-industrial-cho-fed-batch-process 


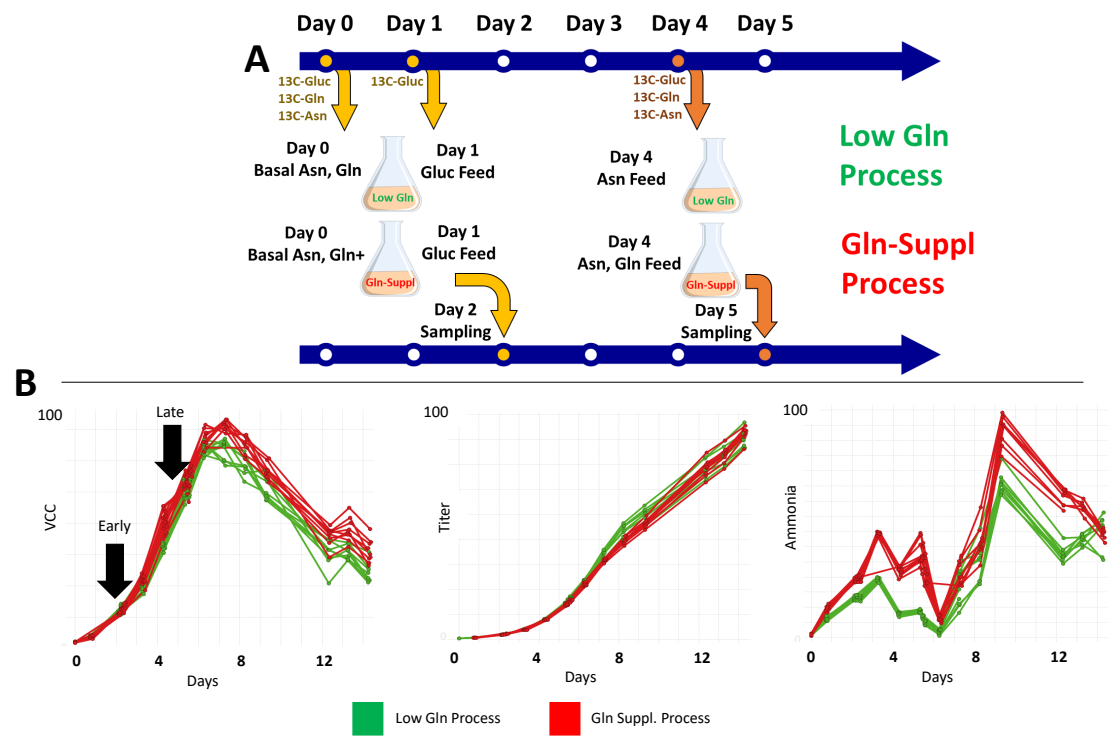

A Early Exponential

Low GIn

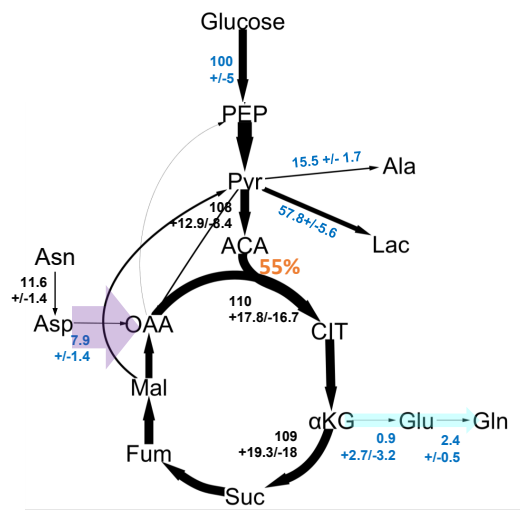

B Early Exponential

GIn Suppl.

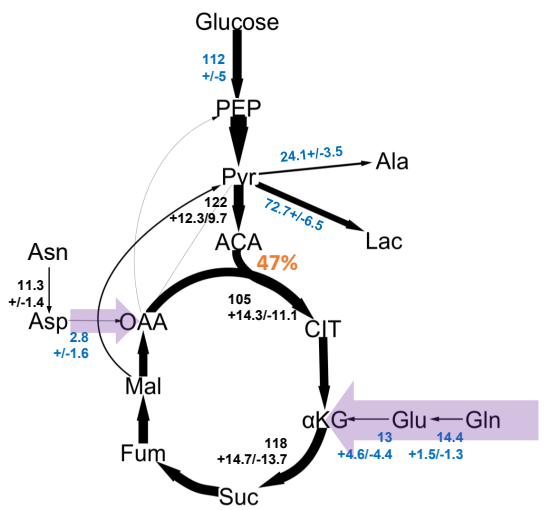

Orange percentages (Fraction of total glucose flux to the TCA cycle)

Schematic flux into the TCA pathway Schematic flux out of the TCA pathway 


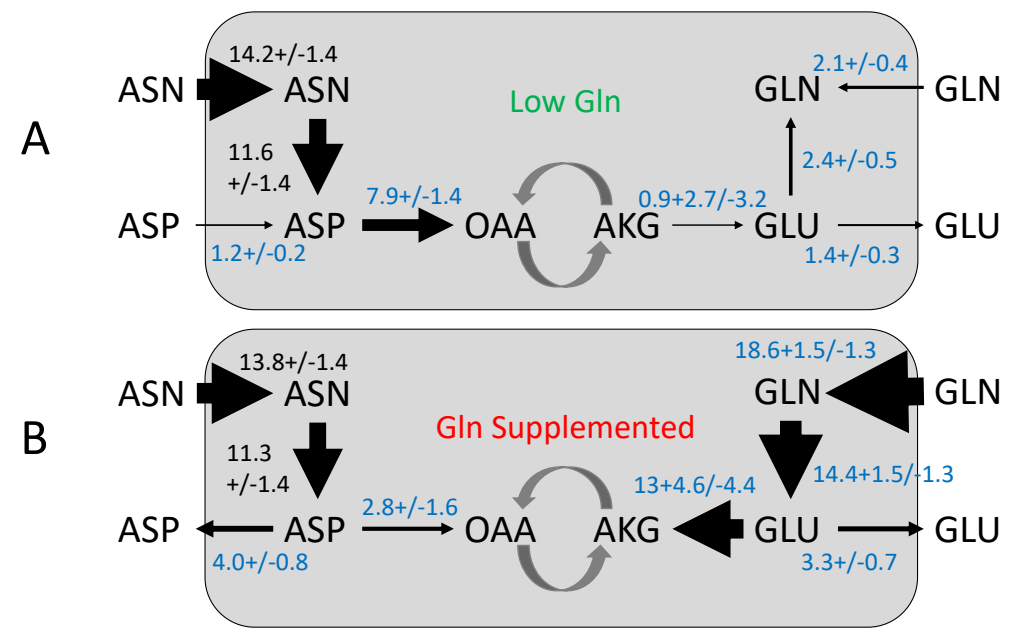

A Late Exponential

Low GIn

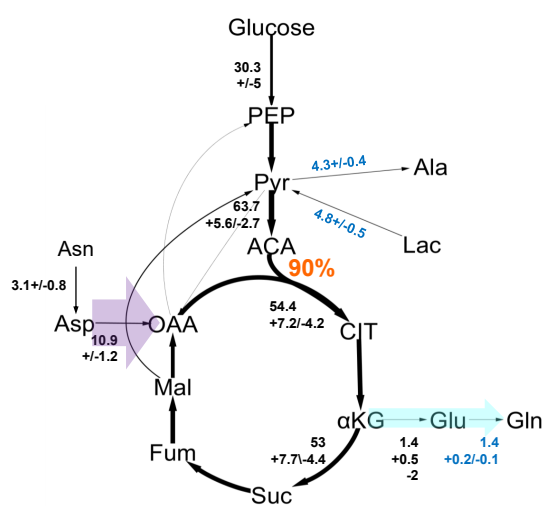

B Late Exponential

GIn Suppl.

Orange percentages (Fraction of total glucose flux to the TCA cycle)

Schematic flux into the TCA pathway

Schematic flux out of the TCA pathway

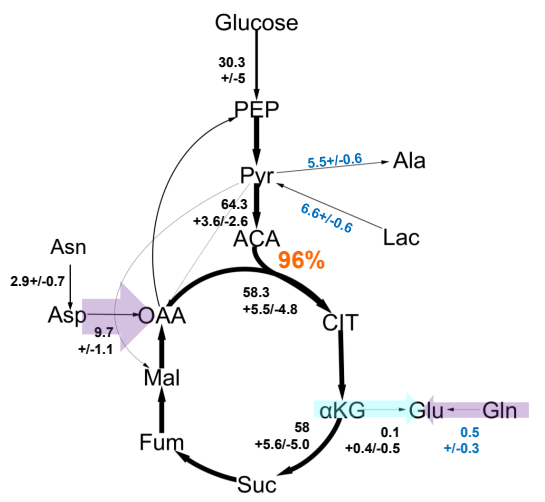



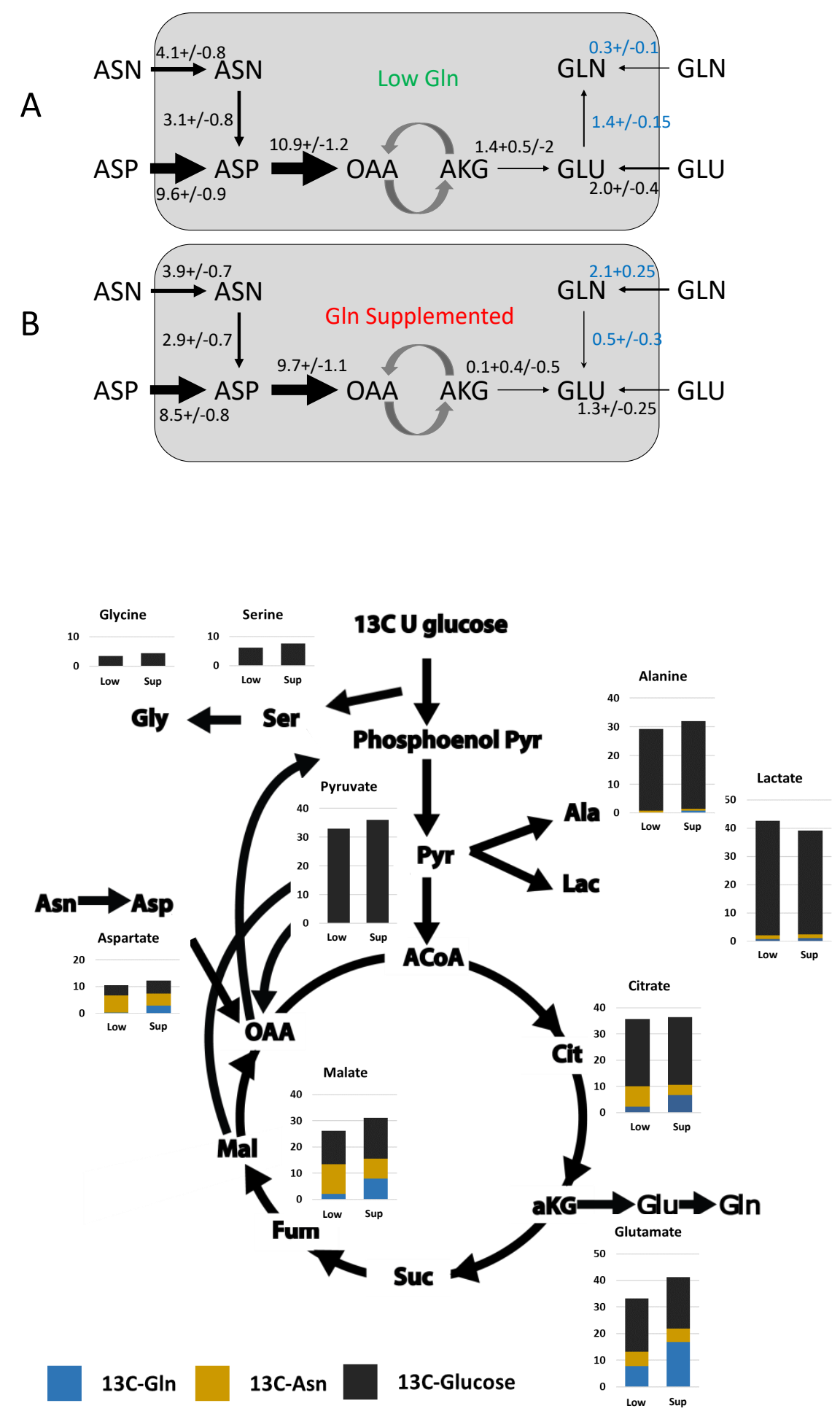

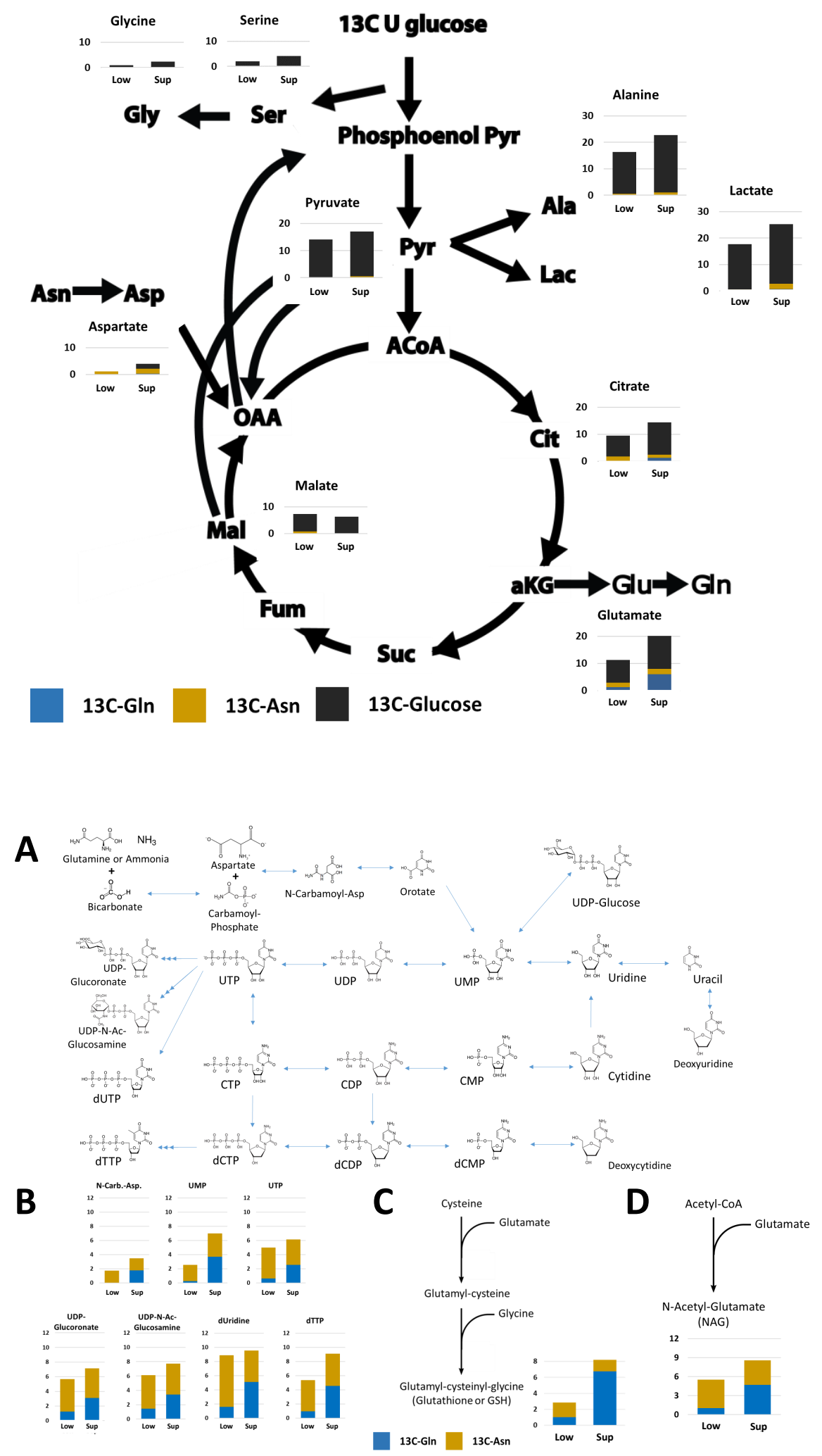